\title{
Catheter Ablation for Persistent and Long-Standing Persistent Atrial Fibrillation
}

\author{
Martin Fiala \\ Department of Cardiology, Center of Cardiovascular Care, Brno, Czech Republic. Department of Internal Medicine and \\ Cardiology, University Hospital, Brno, Czech Republic.
}

\begin{abstract}
Persistent and long-standing persistent atrial fibrillation evolves from complex arrhythmogenic substrate and sources. Multiple studies have shown improved freedom from arrhythmia recurrences if sinus rhythm had been restored during the index ablation; however, such harder procedural endpoint requires laborious stepwise approach almost invariably pursuing non-pulmonary-vein sources. Longer-term conversion of persistent atrial fibrillation into sinus rhythm is associated with significant improvement in major indices of hemodynamic and functional status; these indices also represent major predictors of cardiovascular mortality. Optimal ablation techniques and strategies preserving most of the individual potential for functional improvement need to be established.
\end{abstract}

\section{Introduction}

Catheter ablation for persistent and long-standing persistent atrial fibrillation (LSPAF) is often considered as a low yield endeavor. This is a consequence to generally worse ablation outcome and concerns about detrimental effects of extensive left atrial (LA) or bi-atrial ablation on top of advanced atrial structural remodeling..$^{1-3}$ Serious symptoms including dyspnea, fatigue, and incapacity may be misinterpreted or attributed to aging or other conditions, in particular in the face of preserved left ventricular (LV) systolic function. Quality of life (QoL) associated with persistent AF/LSPAF was previously shown to be poorer than that in paroxysmal $\mathrm{AF}^{4}$ or in other cardiovascular populations, including those with significant coronary artery disease who are older, have worse LV function, and require major intervention. ${ }^{5}$ Growing evidence of significant improvement in LV ejection fraction (LVEF), maximum oxygen consumption at exercise testing $\left(\mathrm{VO}_{2}\right.$ max), and brain natriuretic peptide (BNP) after successful ablation for all persistent $\mathrm{AF}$ types ${ }^{6-9}$ has been sinking in only slowly, although these indices also represent major predictors of cardiovascular mortality. Their amelioration suggests that the ablation impact on survival may be more profound and sooner detectable in high-mortality LSPAF population than in low-mortality patients

\section{Key Words:}

Atrial Fibrillation, Persistent, Long-Standing Persistent, Catheter Ablation.

Disclosures:

None.

Corresponding Author:

Martin Fiala,

Department of Internal Medicine and Cardiology,

University Hospital Brno, Jihlavská 53,

Brno 659 91, Czech Republic. with paroxysmal AF. ${ }^{10}$

This review focuses on efficient abolition of persistent AF substrate and sources and hemodynamic and functional benefit from restored sinus rhythm (SR).

\section{Substrate and Sources of Persistent AF}

The substrate of persistent AF/LSPAF is complex due to advanced atrial dilation and remodeling in terms of scar or amyloid deposits. ${ }^{2,11,12}$ Natural three-dimensional atrial myocardial architecture ${ }^{13}$ modified by structural changes, and modulated by cardiac autonomic nervous system ${ }^{14}$ give rise to heterogeneous AF mechanisms and sources. Localized sources within the antra of pulmonary veins (PV sources) have been widely acknowledged. Localized sources (distinct areas of localized reentry or ectopic foci) outside the PVs remain a matter of dispute. ${ }^{15}$ Participation of macro-reentry circuits (i.e. peri-mitral, roof-dependent, peri-tricuspid) in the background is clear in many $\mathrm{AF}$ instances. Heterogeneous, unstable, and entirely random patterns of activation ${ }^{16,17}$ may also base the AF mechanism, particularly in LSPAF continuous for years.

PV sources and macro-reentry circuits can be effectively abolished by circumferential or linear ablation validated with clear lesionspecific endpoints. In persistent AF; however, such "schematic" ablation was of little efficacy in restoring SR and more extensive lesions were required to achieve this endpoint. ${ }^{18-20}$ Specifically in LSPAF, the immediate impact of PV isolation on AF termination was rather low. ${ }^{21,22}$ In our experience with the last 101 patients ablated for LSPAF, in whom $77 \%$ rate of AF termination and $69 \%$ rate of $\mathrm{SR}$ restoration was attained (Figure 1), left PV isolation terminated AF directly into SR in only 3 patients, and left and right PV isolation converted AF into intermediate atrial tachycardia (AT) in additional 4 and 5 patients, respectively (unpublished data). 


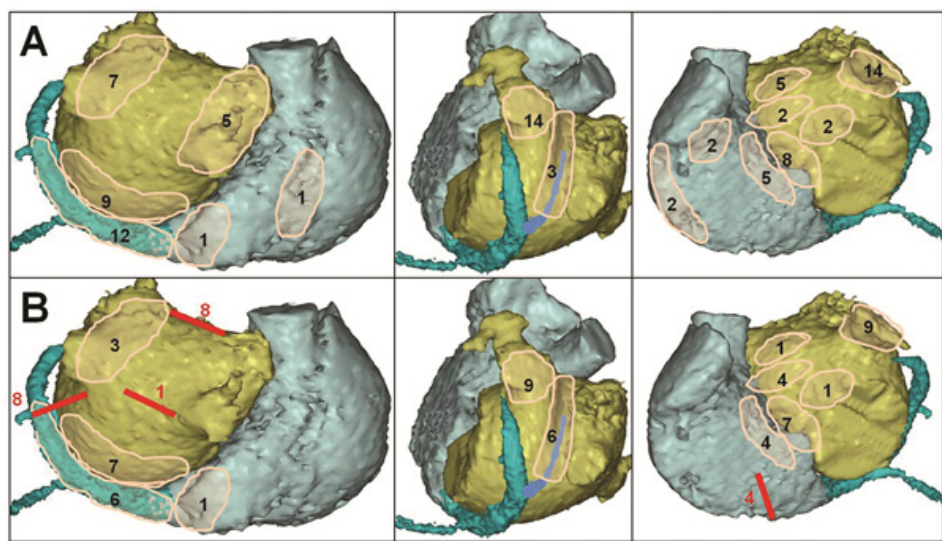

Sites of termination of atrial fibrillation and restoration of sinus Figure 1: rhythm by ablation at the index procedure for long-standing persistent atrial fibrillation

Left-to-right panels show posterior, left lateral, and left anterior oblique views of both atria. Count of events in a specific region is schematically depicted as a number in pink translucent area. Atrial fibrillation was terminated (Panel A) in 78/101 patients: into intermediate atrial tachycardia $(n=68)$, or directly into sinus rhythm $(n=10)$. Direct conversion of atrial fibrillation into sinus rhythm occurred during left pulmonary vein encircling $(n=2)$, at low posterior LA wall along the course of coronary sinus $(n=2)$, during ablation within proximal coronary sinus $(n=2)$, at low left atrial ridge $(n=1)$, left atrial roof $(n=1)$, left-side of limbus of oval fossa $(n=1)$, and right side of limbus of oval fossa $(n=1)$. Sinus rhythm was restored (Panel B) in 70/101 patients, directly from atrial fibrillation in 10 patients (see above) and from intermediate atrial tachycardia in 60 patients. The dominant regions of conversion of atrial fibrillation into sinus rhythm or atrial tachycardia, and of conversion of intermediate atrial tachycardia into sinus rhythm included the low posterior left atrial wall (endocardial aspect of coronary sinus), coronary sinus with ligament/vein of Marshall, left atrial ridge, base of appendage, and interatrial septum. Red lines with counts show instances of sinus rhythm restoration from intermediate peri-mitral (mitral isthmus), roof-dependent (roof or posterior wall), or peri-tricuspid (cavo-tricuspid isthmus) macro-reentry tachycardia/flutter

Gradual AF organization and conversion into one or more types of intermediate AT during systematic stepwise ablation pursuing SR restoration as procedural endpoint support the existence of non-PV localized sources perpetuating persistent AF. In addition, more than half of the intermediate or recurrent ATs arise from localized sources. ${ }^{3,15,18-21,23}$ The non-PV sources may be also present in paroxysmal AF, however, their prevalence is higher in persistent AF/LSPAF, and their number individually increases with the duration of continuous AF to up to the median of 7 in LSPAF. ${ }^{3,24,25}$ Correspondingly, the efficacy in persistent AF termination by selective ablation of the localized sources identified by body-surface panoramic mapping or by possible additional lesions gradually declined with a dramatic shift to worse results in $\mathrm{LSPAF}^{3}$ The location of non-PV sources may depend e.g. on individual structural changes, yet a majority of them cluster at several LA, coronary sinus (CS), and right atrial (RA) regions, ${ }^{3,15,18-21,23}$ which partly overlap the areas with rich autonomic innervation.

In our 101 last patients ablated for LSPAF (see above), the dominant regions of AF conversion into AT/SR or subsequent AT conversion into SR included low posterior LA wall, CS with ligament of Marshall, ridge between left PVs and LA appendage, appendage itself, confluence of left upper PV antrum - LA roof - LA appendage, and inter-atrial septum (Figure 1). Intermediate macro-reentry ATs preceded restoration of SR in 30\% of initial procedures. At first repeat procedure $(n=37)$, AT represented $84 \%$ of the recurrent arrhythmias, and the majority of localized AT sources (either primary recurrent or converted from recurrent $\mathrm{AF}$ by ablation) were found to originate from similar regions along CS/LOM, ridge, appendage and septum.

\section{Ablation Strategies and Endpoints}

Results of different ablation strategies employed in persistent AF/ LSPAF are difficult to compare, and even similar lesion design may be of limited value if immediate ablation impact on the arrhythmia mechanism/sources was diverse. On the contrary, achievement of hard procedural endpoint such as $\mathrm{SR}$ restoration or even subsequent noninducibility of any AF/AT may serve as a more appropriate measure of immediate ablation efficacy independent of specific lesion design or sequence. Strategy consisting of PV isolation followed by direct current cardioversion and subsequent completing of LA roof a mitral isthmus conduction block may miss the non-PV localized sources, and relying on reverse remodeling and dying out of unaffected sources of AF may be conceptually misleading.

The concept of tailored stepwise ablation (Figure 2) was recently doubted by the results of STAR-AF II study, ${ }^{26}$ which was; however, limited by the study protocol not allowing combination of ablation strategies necessary to eliminate all potential AF/AT sources. Correspondingly, the reported efficacy in AF termination (8\%, 22\%, and $45 \%$ for PV isolation, $\mathrm{PV}$ isolation + linear ablation, and PV isolation + complex fractionated electrogram ablation) was low with presumably significantly lower rates of SR restoration (data was not published). The study confirmed only moderate efficacy of PV isolation alone in cure of patients with persistent AF (of whom 21\% were in SR prior to index ablation), and the fact that extensive ablation failing to achieve harder procedural endpoint may be rather proarrhythmic and counterproductive when compared with PV isolation alone. In this respect, some data suggest that the risk of arrhythmia recurrence may be higher in case of extensive stepwise ablation finished with cardioversion from converted AT as compared to extensive ablation finished with cardioversion from AF significantly organized but not converted into AT. ${ }^{27}$

The role of mandatory LA roof and mitral isthmus lines at the index procedure for persistent AF is not clear. Avoiding linear ablation because of concerns about proarrhythmic effect of incomplete lesion may be double-edged. The propensity of remodeled LA for the development or peri-mitral or roof-dependent reentry AT after the index procedure that avoided any linear ablation may not significantly differ from the risk of incomplete or recovered linear lesions. Some data suggested sooner or later need for linear ablation in nearly $90 \%$ of patients to achieve long-term freedom from recurrent AT. ${ }^{28}$

Growing body of evidence favors restoration of SR by the index ablation as an independent predictor of improved long-term rhythm control. The predictors of AF termination included the following variables: shorter duration of continuous $A F,{ }^{29}$ smaller LA size, ${ }^{30}$ shorter long-axis LA diameter, ${ }^{21}$ better LA appendage mechanical function, ${ }^{31,32}$ preserved LVEF, ${ }^{33}$ longer AF cycle length, ${ }^{21,29,30,33,34}$ or lower proportion of high voltage LA sites. ${ }^{21}$

Overall, restoration of $\mathrm{SR}$ at the index ablation for persistent AF/LSPAF - disregard of the sequence of ablation steps - can be interpreted as permanent or temporary suppression of all active AF/ AT sources, and should be pursued as a fundamental procedural endpoint on the way to long-term SR maintenance. Patient-specific ablation with minimum unnecessary damage to the atrial tissue is desired, and may be facilitated by novel body-surface or basketcatheter-based panoramic AF mapping systems.

\section{Long-Term Maintenance of Sinus Rhythm}

Even in case of standardized ablation approach with similar immediate efficacy, outcomes may be difficult to compare between persistent AF populations disparate in terms of underlying atrial structural remodeling ${ }^{2,35}$ or clinical characteristics such as duration of 


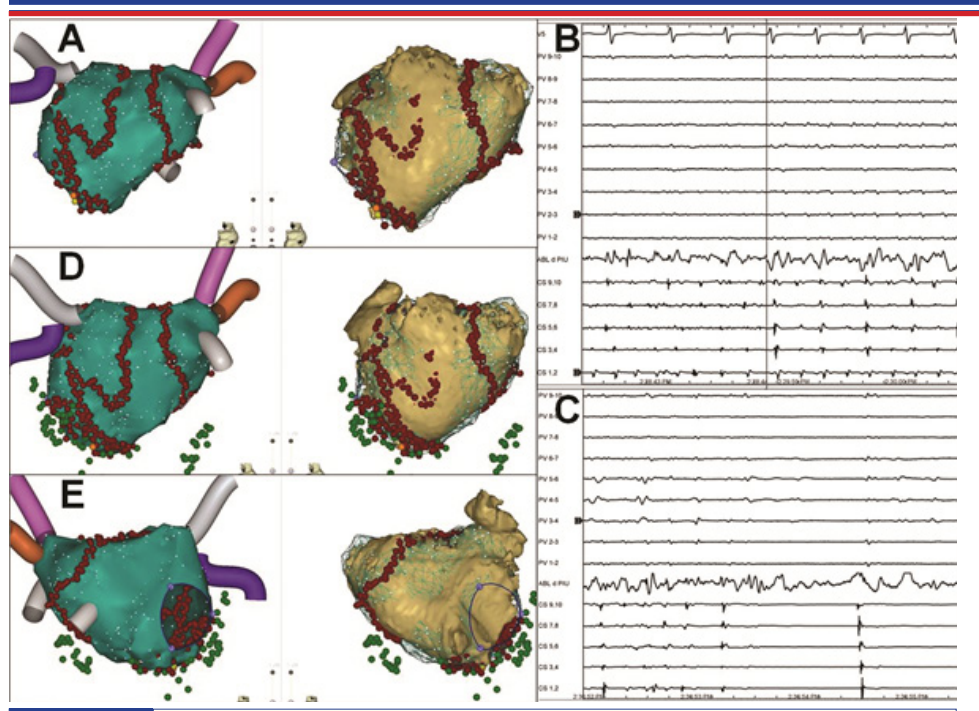

Figure 2: Example of stepwise ablation with mandatory pulmonary vein isolation as the first step

Posterior (panels A,D) and anterior (panel E) view of electroanatomic (left) and computed tomography (right) left atrial maps. Following pulmonary vein isolation, atrial fibrillation was converted into transient atrial tachycardia (Panels $A, B$ ) and subsequently into sinus rhythm (Panels A,C) (yellow and orange dots) by electrogram-guided ablation targeting the low posterior left atrial wall (endocardial aspect of coronary sinus). Mitral isthmus, left atrial roof, and cavotricuspid isthmus conduction blocks with coronary sinus isolation (green dots) were completed after restoration of sinus rhythm (Panel D,E). Noninducibility of any atrial tachyarrhythmia was eventually confirmed by atrial pacing up to $300 \mathrm{bpm}$, isoproterenol, and adenosine. No septal, left atrial anterior wall or appendage ablation was required in this case (see anterior view of the left atrium in Panel E), and timely left atrial appendage activation was preserved along with restoration of the appendage outflow velocity from peak $0.42 \mathrm{~m} / \mathrm{s}$ during atrial fibrillation to 0.66 $\mathrm{m} / \mathrm{s}$ during sinus rhythm. Patient's / procedure characteristics: male, age 60 years, $108 \mathrm{~kg}, 196$ $\mathrm{cm}$, continuous atrial fibrillation for $\mathbf{2 4}$ months, history of temporary hypothyroidism induced by amiodarone, prior failure to convert atrial fibrillation by direct current cardioversion, left atrial volume by the CARTO system $170 \mathrm{ml}$, procedure, fluoroscopy, and radiofrequency energy delivery times 270,11 , and 120 minutes respectively

continuous $\mathrm{AF}$ or resistance to antiarrhythmic drugs before ablation.

Freedom from AF/AT after single ablation as the hardest outcome measure is commonly low, but can be markedly improved by repeat ablation..$^{21,36-38} \mathrm{PV}$ isolation alone was associated with only moderate AF/AT-free outcome in patients with persistent $\mathrm{AF}^{26}$ or LSPAF. ${ }^{39}$ "Good arrhythmia control" as a more clinically relevant outcome measure is usually favorable and can reach acceptable $~ 90 \%$ after stepwise/repeat ablation. ${ }^{21,36,37}$ Most of repeat procedures are usually performed within the first two years, although late AT recurrences due to evolving substrate may gradually reduce the long-term outcome and require delayed repeat ablation. ${ }^{37,38}$ Of our last 101 patients with LSPAF and 69\% SR restoration rate at the index procedure (see above), $92 \%$ patients remain in stable SR at the end of $49 \pm 23$ month follow-up and after 1.4 procedures/patient ( $84 \%$ were off class I/III AADs; $81 \%$ were free from any AF/AT recurrence after the last ablation).

Pre-procedural factors of better AF/AT-free survival after single or repeat ablation for persistent AF/LSPAF included smaller LA size, ${ }^{36,40}$ shorter continuous AF duration, ${ }^{21,29,36,39-41}$ younger age, ${ }^{30,42}$ male gender, ${ }^{40}$ absence of congestive heart failure, ${ }^{40}$ absence of hypertension, ${ }^{30}$ higher LA appendage outflow velocity, ${ }^{21,32}$ LA systolic strain and LAA wall velocity ${ }^{44}$ or AF cycle length. ${ }^{21,29,30,45}$ Restoration of SR at the index ablation appeared as a powerful predictor of outcome in multiple studies. . $1227,30,32,33,36-38,40,41,44^{\text {The }}$ predictive value of restored SR at the initial ablation was shown to extend beyond repeat procedures. ${ }^{21,30,40,44}$

Noninducibility of AF/AT, suggesting non-transient effect on distinct AF/AT source(s), is intuitively superior to simple restoration of SR. Although unrealistic in many initial procedures, noninducibility is feasible in a majority of re-do cases. We have recently demonstrated the predictive value of AF/AT noninducibility at repeat ablation for long-term arrhythmia-free outcome in patients with LSPAF; when it was not attained despite termination of recurrent AF/AT, the outcome was significantly worse. ${ }^{21}$

Overall, achievement of SR restoration by ablation and subsequent arrhythmia noninducibility serve as more reliable procedural endpoints; however, do not guarantee 100\% arrhythmia-free outcome, because no current tool can discern between the desired ablation -induced tissue necrosis (future scar) and injured but surviving myocardium that may recover to resumption of existing $\mathrm{AF} / \mathrm{AT}$ source(s) or proarrhythmic effect, in addition to the natural disease progression and evolution of new AF/AT sources.

Hemodynamic and Functional Benefits from Successful Ablation

Improvement in QoL has been emphasized as the major incentive for catheter ablation of paroxysmal AF. Insidiously progressive symptoms of LSPAF have been recently shown to impair baseline QoL even more than episodic palpitations related to paroxysmal $\mathrm{AF}^{4}$ Consequently, the magnitude of improvement 3 years after the index ablation was greater in patients with LSPAF, which resulted in comparable absolute post-ablation QoL levels in both paroxysmal AF and LSPAF populations. ${ }^{4}$ In addition to QoL improvement, several recent studied assessed the hemodynamic and functional effects of catheter ablation for persistent AF and LSPAF in greater detail. Two randomized studies demonstrated superiority of catheter ablation over medical rate control in the subjects with persistent $\mathrm{AF}$ and $\mathrm{LV}$ systolic dysfunction. ${ }^{6,7}$ These studies reported consistent post-ablation $\mathrm{VO}_{2}$ max improvement contrasting with further deterioration on rate control therapy. Post-ablation LVEF significantly improved in both studies, compared with borderline increase or further significant decrease in the rate control arms. ${ }^{6,7}$ The magnitude of improvement in BNP and Minnesota Living with Heart Failure Questionnaire Score was significant in ablated patients, but less prominent or insignificant in patients with rate control. , $^{7}$

The benefits in heart failure patients are not surprising given the LV systolic function is frequently derpessed by the arrhythmia itself. There is a paucity of data on functional benefit in non-heart

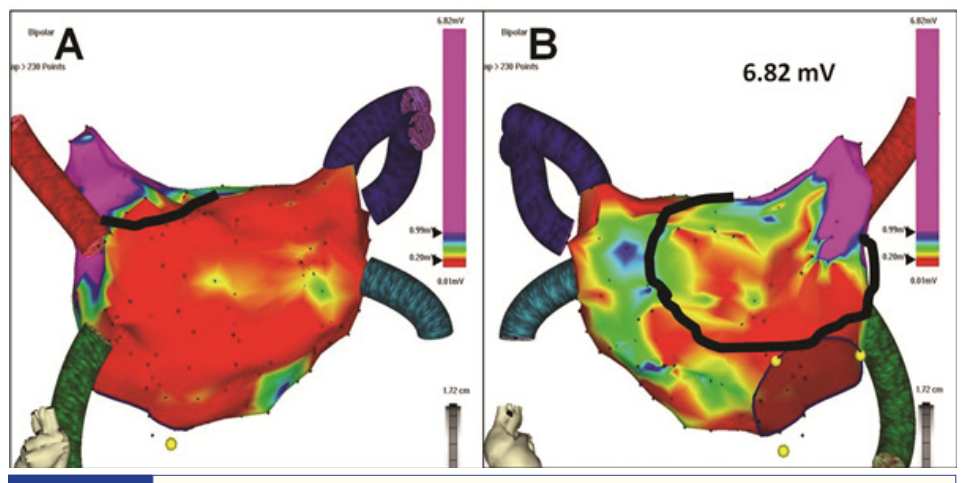

Figure 3:

Characteristic pre-ablation voltage pattern in long-standing persistent atrial fibrillation

Prior to any ablation, the electroanatomic left atrial mapping $(\mathrm{A}=$ posterior view, $\mathrm{B}=$ anterio view) reveals low voltage $(\leq 0.2 \mathrm{mV}$ coded in red color) across the whole posterior wall, while favorable voltage ( $\geq 1 \mathrm{mV}$ coded in purple color) is present in the appendage (maximum voltage $6.82 \mathrm{mV}$ ). Black line circumscribes the amount of surviving atrial myocardium that would be lost as a result of indiscriminate appendage isolation and complete anterior linear lesion with possible consequences for global left atrial transport function 
failure patients. We have recently found a significant post-ablation improvement in $\mathrm{VO}_{2}$ max, LVEF, NT-proBNP, and QoL in 160 patients (including 20\% with baseline LVEF $\leq 40 \%$ ) undergoing catheter ablation for LSPAF resistant to amiodarone therapy and cardioversion. ${ }^{8}$ In another study, patients with LSPAF designated as asymptomatic experienced a significant post-ablation improvement in $\mathrm{VO}_{2} \max (+3.7 \pm 10 \mathrm{~mL} / \mathrm{kg} / \mathrm{min})$ and in physical component score, which was not present in patients with failed procedures. ${ }^{9}$ The study included $~ 13 \%$ patients with the diagnosis of congestive heart failure, which implied that the selection of patients based on symptoms may not exclude subjects who got used to live with AF. ${ }^{9}$ To address a more homogenous LSPAF population with preserved LV systolic function, we have compared functional benefits in 155 LSPAF patients with no prior history of $\mathrm{LV}$ systolic dysfunction (i.e. LVEF consistently $250 \%$ ) vs. 41 LSPAF patients with LVEF consistently $\leq 40 \%$ for $23 \pm 20$ pre-ablation months (unpublished data). In the latter group,LVEF increased 12 months after index ablation by $22 \pm 8 \%$ and $85 \%$ of these patients experienced LVEF normalization to $\geq 50 \%$. The magnitude of $\mathrm{VO}_{2} \max$ improvement was $3.0 \pm 4.4$ in patients with $\mathrm{LVEF} \geq 50 \%$ vs. $3.9 \pm 5.1 \mathrm{~mL} / \mathrm{kg} / \mathrm{min}$ in patients with $\mathrm{LVEF} \leq 40 \%$. Of note, near two-fold elevation of NT-proBNP over the pathological cut-off value $(942 \pm 625 \mathrm{pg} / \mathrm{mL})$ and its subsequent 12 -month post-ablation decrease by $711 \pm 562 \mathrm{pg} /$ $\mathrm{mL}$ in patients with normal baseline $\mathrm{LV}$ function corroborated severe adverse hemodynamic effects of LSPAF also in this population.

In summary, recent data show a significant hemodynamic and functional benefit from SR restored by successful catheter ablation of persistent AF/LSPAF. Limited data suggests that the LSPAFrelated loss of exercise capacity $\left(\mathrm{VO}_{2} \max \right)$ corresponds to at least $3 \mathrm{~mL} / \mathrm{kg} / \mathrm{min}$ even in asymptomatic and/or non-heart failure patients. Elevated (NT-pro)BNP might help the clinicians to attribute insidiously progressive dyspnea, fatigue, and incapacity to the arrhythmia itself and advice the patients about catheter ablation.

\section{Left Atrial Appendage - Sacrifice or Protect?}

A significant proportion of non-PV sources maintaining persistent AF/LSPAF are located within the LA appendage and abutting ridge and ligament of Marshall. Selective ablation of these sources is laborious and has to be often repeated. Isolation of these sources by peri-ostial LA appendage ablation, ${ }^{46}$ or by LA anterior line supplementing complete left PV isolation and mitral isthmus and LA roof lines ${ }^{47}$ without doubt improves ablation efficacy; however, possible adverse effects of these seductive steps have yet to be understood. Increased thromboembolic risk of electrically isolated non-contracting LA appendage has been already shown. ${ }^{47}$ Appendage occlusion/exclusion may rectify this adverse effect, but does not solve possible hemodynamic consequences. It is known that the contribution of active LA transport to the LV filling increases with exertion to up to $40 \%$. It is also obvious that severe structural remodeling in patients with persistent AF/LSPAF typically involves the main LA body and specifically the posterior wall, while the appendage as the only trabecular LA structure seems to be better protected against major myocyte changes and interstitial fibrosis ${ }^{48}$ (Figure 3). Consequently, LA appendage mechanical depression due to $\mathrm{AF}$ can reverse upon restoration of $\mathrm{SR},{ }^{8,49,50}$ and its relative contribution to the active LA transport and LV filling may be greater as compared to subjects with healthy LA. ${ }^{51}$ Therefore, restored LAA emptying and resulting favorable post-ablation active trans-mitral flow may be of particular importance for global hemodynamic and functional status particularly in active subjects without major comorbidities. Some surgical studies suggested adverse effects of the LA appendage exclusion on the LA transport function. ${ }^{52,53}$

In addition to complete LA appendage isolation, the consequences of LA appendage activation/outflow delay (i.e. partial or complete appendage emptying against closed mitral valve) are even less well understood. Detrimental effects of the LA anterior line on the LA transport function have been recently challenged; however, the study did not address the issue adequately by failing to document the appendage outflow delay in a majority of patients at follow-up. ${ }^{54}$ We have recently found association between delayed LA appendage outflow (interval between the onset of LA appendage outflow and the onset of QRS complex $<50 \mathrm{~ms}$ on 12 month post-ablation transesophageal echocardiography) and lesser increase in the LA appendage outflow velocity ( $7 \pm 25$ vs. $20 \pm 18 \mathrm{~cm} / \mathrm{s} ; \mathrm{P}<0.00008)$ or lower peak $A$ wave velocity of trans-mitral flow $(35 \pm 11$ vs. $39 \pm 12$ $\mathrm{cm} / \mathrm{s} ; \mathrm{P}=0.04$ ) as compared to patients with early appendage outflow (interval between the onset of LA appendage outflow and QRS complex $\geq 50 \mathrm{~ms}$ ). Moreover, when relative improvements in $\mathrm{VO}_{2} \mathrm{max}$, LVEF, NT-proBNP, and EQ-5D were summed into a composite score reflecting change in global hemodynamic and functional status,
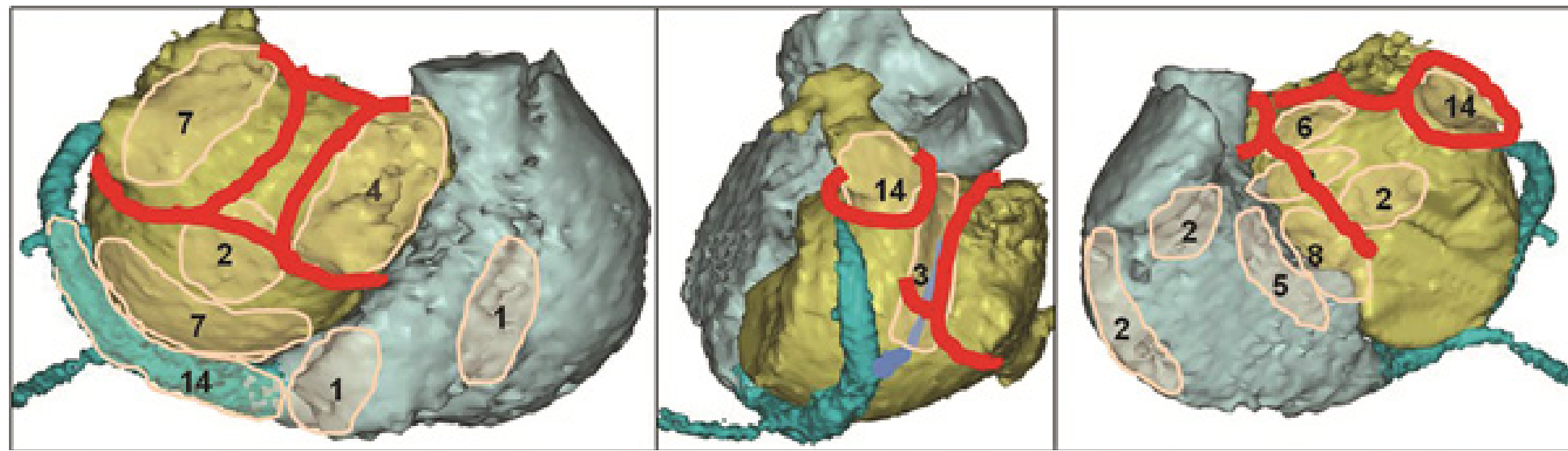

Figure 4:

Schematic depiction of common lesions / appendage exclusion during thoracoscopic epicardial ablation

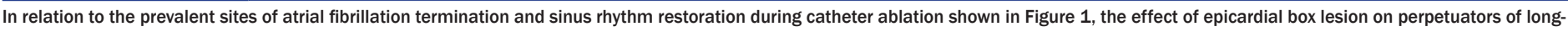

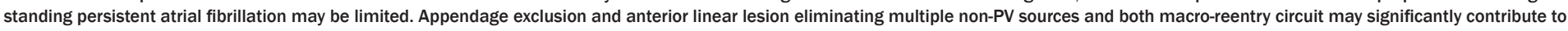

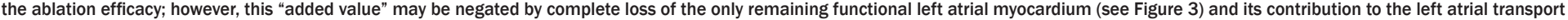
function. Non-pulmonary vein sources located within the coronary sinus and inter-atrial septum are not suitable or accessible to the epicardial radiofrequency ablation 
the LA appendage outflow delay (along with older age, female gender, continued antiarrhythmic drugs, and lower LA appendage outflow velocity) was adversely related to the improvement in this score. ${ }^{55}$

Overall, restoration of AF-related LA appendage mechanical dysfunction in patients with persistent AF/LSPAF may have undervalued salutary effects on global LA transport of blood and global functional status, and may warrant a more selective approach to the AF/AT sources originating within the LA appendage. More studies are needed to assess the hemodynamic role of the LA appendage specifically in severely structurally remodelled LA.

\section{Surgical (Hybrid) vs. Catheter Ablation}

Efforts and expertise unavoidable for successful systematic catheter ablation in patients with persistent AF/LSPAF spurred on proliferation of "hybrid" thoracoscopic epicardial and endocardial catheter ablation performed either simultaneously or in sequence. In the face of increased risks of serious complications, true advantages of thoracoscopic epicardial ablation should be confronted with true reasons or sources that underlie the efficacy of hybrid approach. In the context with distribution of sites where LSPAF and subsequent intermediate ATs were terminated by catheter ablation (shown in Figure 1), mere epicardial radiofrequency box lesion seems to be of limited "added value", because it misses a majority of non-PV sources (Figure 4). True advantages of epicardial ablation may include direct destruction of ligament of Marshall and epicardial approach to thick myocardial structures (e.g. LA roof) or ganglionic plexi (although recent AFACT trial presented at Cardiostim/Nice 2016 showed no further improvement in outcome after extra ganglionic plexi ablation at the expense of increased rate of complications). The efficacy of "hybrid" approach most likely consists in the LA appendage exclusion and ablation of LA anterior wall; (non)inclusion of these steps appeared to be responsible for diverse outcomes in multiple studies. ${ }^{56,57}$ These steps can eliminate frequent localized sources and both LA macro-reentry circuits; however, at a price of loss of the only remaining contractile LA structures with unclear impact on functional status specifically in younger active subjects (see above, Figures 3,4).

Most importantly, thoracoscopic approach has been associated with high prevalence of serious complications including early postprocedural death. ${ }^{58,59}$ Simultaneous surgical and catheter ablation will always struggle with peri-procedural anticoagulation and balance between the risk of thromboembolism and bleeding. Finally, sequential hybrid ablation automatically means two procedures per patient, with possible further repeat procedures in long-term followup.

Overall, the major principal limitations of thoracoscopic epicardial ablation include inaccessibility to many non-PV sources within the septum or inability to safely apply radiofrequency energy to the CS sources, and hence failure to cure a high proportion of LSPAF patients with a single procedure. In addition, "schematic" surgical ablation unable to individualize patient-specific lesions may result in unnecessary destruction of atrial tissue responsible for hemodynamic and functional benefit from restored SR. Such a concept may relieve the electrophysiologists of tedious systematic search for AF sources, but goes against the quest for more selective ablation and maximized functional improvement.

\section{Conclusions}

Ablation for persistent AF and LSPAF evolves amid the gaps in our understanding of the arrhythmia mechanisms and sources, their evolution in timeline and detection by mapping, and fine balance between ablation safety and efficacy. Significant hemodynamic and functional effects of successful catheter ablation may reflect the magnitude of reversible hemodynamic and functional impairment related to the arrhythmia itself. This functional deterioration, which is often underrated by the clinical judgement, may insidiously progress into heart failure and increased mortality that has not improved over the last 20 years despite nearly optimal anticoagulation. Therefore, functional and potential mortality benefits may encourage catheter ablation of persistent AF/LSPAF in high proportion of patients up to now destined to lifelong rate control and oral anticoagulation. An increasing body of evidence indicates that favorable outcome requires elimination of all arrhythmic sources including the sources of intermediate AT; however, such procedures are laborious and lengthy with current mapping and ablation tools. Therefore, novel mapping and ablation technologies along with further insight into the AF pathophysiology are desired to facilitate faster selective abolition of non-PV sources, and by minimizing unnecessary atrial myocardial damage allow maximum individual functional benefit from restored SR.

\section{References}

1. Cappato Riccardo, CalkinsHugh, ChenShih-Ann, DaviesWyn, IesakaYoshito, KalmanJonathan, KimYou-Ho, KleinGeorge, NataleAndrea, PackerDouglas, SkanesAllan, AmbrogiFederico, BiganzoliElia. Updated worldwide survey on the methods, efficacy, and safety of catheter ablation for human atrial fibrillation. Circ Arrhythm Electrophysiol. 2010;3 (1):32-8.

2. McGann Christopher, AkoumNazem, PatelAmit, KholmovskiEugene, ReveloPatricia, DamalKavitha, WilsonBrent, CatesJosh, HarrisonAlexis, RanjanRavi, BurgonNathan S, GreeneTom, KimDan, DibellaEdward V R, ParkerDennis, MacleodRob S, MarroucheNassir F. Atrial fibrillation ablation outcome is predicted by left atrial remodeling on MRI. Circ Arrhythm Electrophysiol. 2014;7 (1):23-30.

3. Haissaguerre Michel, HociniMeleze, DenisArnaud, ShahAshok J, KomatsuYuki, YamashitaSeigo, DalyMatthew, AmraouiSana, ZellerhoffStephan, PicatMarieQuitterie, QuotbAdam, JeselLaurence, LimHan, PlouxSylvain, BordacharPierre, AttuelGuillaume, MeilletValentin, RitterPhilippe, DervalNicolas, SacherFrederic, BernusOlivier, CochetHubert, JaisPierre, DuboisRemi. Driver domains in persistent atrial fibrillation. Circulation. 2014;130 (7):530-8.

4. Bulková Veronika, FialaMartin, HavránekStěpán, SimekJan, SkňouřilLibor, JanuškaJaroslav, SpinarJindřich, WichterleDan. Improvement in quality of life after catheter ablation for paroxysmal versus long-standing persistent atrial fibrillation: a prospective study with 3-year follow-up. J Am Heart Assoc. 2014;3 (4): $\mathrm{e} 000881$.

5. Dorian P,JungW, NewmanD, PaquetteM, WoodK, AyersG M, CammJ, AkhtarM, LuderitzB. The impairment of health-related quality of life in patients with intermittent atrial fibrillation: implications for the assessment of investigational therapy. J. Am. Coll. Cardiol. 2000;36 (4):1303-9.

6. Jones David G, HaldarShouvik K, HussainWajid, SharmaRakesh, FrancisDarrel P, Rahman-HaleyShelley L, McDonaghTheresa A, UnderwoodS Richard, MarkidesVias, WongTom. A randomized trial to assess catheter ablation versus rate control in the management of persistent atrial fibrillation in heart failure. J. Am. Coll. Cardiol. 2013;61 (18):1894-903.

7. Hunter Ross J, BerrimanThomas J, DiabIhab, KamdarRavindu, RichmondLaura, BakerVictoria, GoromonziFarai, SawhneyVinit, DuncanEdward, PageStephen P, UllahWaqas, UnsworthBeth, MayetJamil, DhinojaMehul, EarleyMark J, SportonSimon, SchillingRichard J. A randomized controlled trial of catheter ablation versus medical treatment of atrial fibrillation in heart failure (the 
CAMTAF trial). Circ Arrhythm Electrophysiol. 2014;7 (1):31-8.

8. Fiala Martin, WichterleDan, BulkováVeronika, SknourilLibor, NevralováRenáta, TomanOndrej, DordaMiloslav, JanuskaJaroslav, SpinarJindrich. A prospective evaluation of haemodynamics, functional status, and quality of life after radiofrequency catheter ablation of long-standing persistent atrial fibrillation. Europace. 2014;16 (1):15-25.

9. Mohanty Sanghamitra, SantangeliPasquale, MohantyPrasant, Di BiaseLuigi, HolcombShawna, TrivediChintan, BaiRong, BurkhardtDavid, HongoRichard, HaoSteven, BeheirySalwa, SantoroFrancesco, ForleoGiovanni, GallinghouseJoseph G, HortonRodney, SanchezJavier E, BaileyShane, HranitzkyPatrick M, ZagrodzkyJason, NataleAndrea. Catheter ablation of asymptomatic longstanding persistent atrial fibrillation: impact on quality of life, exercise performance, arrhythmia perception, and arrhythmia-free survival. J. Cardiovasc. Electrophysiol. 2014;25 (10):1057-64.

10. Lip Gregory Y H, LarocheCécile, IoachimPopescu Mircea, RasmussenLars Hvilsted, Vitali-SerdozLaura, PetrescuLucian, DarabantiuDan, CrijnsHarry J G M, KirchhofPaulus, VardasPanos, TavazziLuigi, MaggioniAldo P, BorianiGiuseppe. Prognosis and treatment of atrial fibrillation patients by European cardiologists: one year follow-up of the EURObservational Research Programme-Atrial Fibrillation General Registry Pilot Phase (EORP-AF Pilot registry). Eur. Heart J. 2014;35 (47):3365-76.

11. Fiala Martin, WichterleDan, ChovancíkJan, BulkováVeronika, WojnarováDorota, NevralováRenáta, JanuskaJaroslav. Left atrial voltage during atrial fibrillation in paroxysmal and persistent atrial fibrillation patients. Pacing Clin Electrophysiol. 2010;33 (5):541-8.

12. Goette Andreas, RöckenChristoph. Atrial amyloidosis and atrial fibrillation: a gender-dependent "arrhythmogenic substrate"?. Eur. Heart J. 2004;25 (14):11856.

13. Ho S Y, Sanchez-QuintanaD, CabreraJ A, AndersonR H. Anatomy of the left atrium: implications for radiofrequency ablation of atrial fibrillation. J. Cardiovasc. Electrophysiol. 1999;10 (11):1525-33.

14. Nakagawa Hiroshi, ScherlagBenjamin J, PattersonEugene, IkedaAtsuhsi, LockwoodDeborah, JackmanWarren M. Pathophysiologic basis of autonomic ganglionated plexus ablation in patients with atrial fibrillation. Heart Rhythm. 2009;6 (12 Suppl):S26-34.

15. Haïssaguerre Michel, HociniMélèze, SandersPrashanthan, TakahashiYoshihide, RotterMartin, SacherFrederic, RostockThomas, HsuLi-Fern, JonssonAnders, O'NeillMark D, BordacharPierre, ReuterSylvain, RoudautRaymond, ClémentyJacques, JaïsPierre. Localized sources maintaining atrial fibrillation organized by prior ablation. Circulation. 2006;113 (5):616-25.

16. MOE G K, RHEINBOLDTW C, ABILDSKOVJ A. A COMPUTER MODEL OF ATRIAL FIBRILLATION. Am. Heart J. 1964;67:200-20.

17. Lee Geoffrey, KumarSaurabh, TehAndrew, MadryAndrew, SpenceSteven, LarobinaMarco, GoldblattJohn, BrownRobin, AtkinsonVictoria, MotenSimon, MortonJoseph B, SandersPrashanthan, KistlerPeter M, KalmanJonathan M. Epicardial wave mapping in human long-lasting persistent atrial fibrillation: transient rotational circuits, complex wavefronts, and disorganized activity. Eur. Heart J. 2014;35 (2):86-97.

18. Haïssaguerre Michel, SandersPrashanthan, HociniMélèze, TakahashiYoshihide, RotterMartin, SacherFrederic, RostockThomas, HsuLi-Fern, BordacharPierre, ReuterSylvain, RoudautRaymond, ClémentyJacques, JaïPierre. Catheter ablation of long-lasting persistent atrial fibrillation: critical structures for termination. J. Cardiovasc. Electrophysiol. 2005;16 (11):1125-37.

19. Rostock Thomas, StevenDaniel, HoffmannBoris, ServatiusHelge, DrewitzImke, SydowKarsten, MüllerleileKai, VenturaRodolfo, WegscheiderKarl, MeinertzThomas, WillemsStephan. Chronic atrial fibrillation is a biatrial arrhythmia: data from catheter ablation of chronic atrial fibrillation aiming arrhythmia termination using a sequential ablation approach. Circ Arrhythm
Electrophysiol. 2008;1 (5):344-53.

20. Fiala Martin, ChovancíkJan, NevralováRenáta, NeuwirthRadek, JiravskýOtakar, JanuskaJaroslav, BrannyMarian. Termination of long-lasting persistent versus short-lasting persistent and paroxysmal atrial fibrillation by ablation. Pacing Clin Electrophysiol. 2008;31 (8):985-97.

21. Fiala Martin, BulkováVeronika, ŠkňouřilLibor, NevřalováRenáta, TomanOndřej, JanuškaJaroslav, ŠpinarJindřich, WichterleDan. Sinus rhythm restoration and arrhythmia noninducibility are major predictors of arrhythmia-free outcome after ablation for long-standing persistent atrial fibrillation: a prospective study. Heart Rhythm. 2015;12 (4):687-98.

22. Elayi Claude S, Di BiaseLuigi, BarrettConor, ChingChi Keong, al AlyMoataz, LucciolaMaria, BaiRong, HortonRodney, FahmyTamer S, VermaAtul, KhaykinYaariv, Shahjignesh, MoralesGustavo, HongoRichard, HaoSteven, BeheirySalwa, ArrudaMauricio, SchweikertRobert A, CummingsJennifer, BurkhardtJ David, WangPaul, Al-AhmadAmin, CauchemezBruno, GaitaFiorenzo, NataleAndrea. Atrial fibrillation termination as a procedural endpoint during ablation in long-standing persistent atrial fibrillation. Heart Rhythm. 2010;7 (9):1216-23.

23. Rostock Thomas, DrewitzImke, StevenDaniel, HoffmannBoris A, SalukheTushar V, BockKarsten, ServatiusHelge, AydinMuhammet Ali, MeinertzThomas, WillemsStephan. Characterization, mapping, and catheter ablation of recurrent atrial tachycardias after stepwise ablation of long-lasting persistent atrial fibrillation. Circ Arrhythm Electrophysiol. 2010;3 (2):160-9.

24. Zhao Yonghui, Di BiaseLuigi, TrivediChintan, MohantySanghamitra, BaiRong, MohantyPrasant, GianniCarola, SantangeliPasquale, HortonRodney, SanchezJavier, GallinghouseG Joseph, ZagrodzkyJason, HongoRichard, BeheirySalwa, LakkireddyDhanunjaya, ReddyMadhu, HranitzkyPatrick, AlAhmadAmin, ElayiClaude, BurkhardtJ David, NataleAndrea. Importance of nonpulmonary vein triggers ablation to achieve long-term freedom from paroxysmal atrial fibrillation in patients with low ejection fraction. Heart Rhythm. 2016;13 (1):141-9.

25. Narayan Sanjiv M, KrummenDavid E, ShivkumarKalyanam, CloptonPaul, RappelWouter-Jan, MillerJohn M. Treatment of atrial fibrillation by the ablation of localized sources: CONFIRM (Conventional Ablation for Atrial Fibrillation With or Without Focal Impulse and Rotor Modulation) trial.J. Am. Coll. Cardiol. 2012;60 (7):628-36.

26. Verma Atul, JiangChen-yang, BettsTimothy R, ChenJian, DeisenhoferIsabel, MantovanRoberto, MacleLaurent, MorilloCarlos A, HaverkampWilhelm, WeerasooriyaRukshen, AlbenqueJean-Paul, NardiStefano, MenardiEndrj, NovakPaul, SandersPrashanthan. Approaches to catheter ablation for persistent atrial fibrillation. N. Engl. J. Med. 2015;372 (19):1812-22.

27. Ammar Sonia, HesslingGabriele, ReentsTilko, PaulikMaria, FichtnerStephanie, SchönPatrick, DillierRoger, KathanSusanne, JilekClemens, KolbChristof, HallerBernhard, DeisenhoferIsabel. Importance of sinus rhythm as endpoint of persistent atrial fibrillation ablation. J. Cardiovasc. Electrophysiol. 2013;24 (4):388-95.

28. Knecht Sébastien, HociniMélèze, WrightMatthew, LelloucheNicolas, O’NeillMark D, MatsuoSeiichiro, NaultIsabelle, ChauhanVijay S, MakatiKevin J, BevilacquaMichela, LimKang-Teng, SacherFrederic, DeplagneAntoine, DervalNicolas, BordacharPierre,JaïsPierre,ClémentyJacques, HaïssaguerreMichel. Left atrial linear lesions are required for successful treatment of persistent atrial fibrillation. Eur. Heart J. 2008;29 (19):2359-66.

29. Matsuo Seiichiro, LelloucheNicolas, WrightMatthew, BevilacquaMichela, KnechtSébastien, NaultIsabelle, LimKang-Teng, ArantesLeonardo, O’NeillMark D, PlatonovPyotr G, CarlsonJonas, SacherFrederic, HociniMélèze, JaïsPierre, HaïssaguerreMichel. Clinical predictors of termination and clinical outcome of catheter ablation for persistent atrial fibrillation. J. Am. Coll. Cardiol. 2009;54 (9):788-95. 
30. Heist E Kevin, ChalhoubFadi, BarrettConor, DanikStephan, RuskinJeremy N, MansourMoussa. Predictors of atrial fibrillation termination and clinical success of catheter ablation of persistent atrial fibrillation. Am. J. Cardiol. 2012;110 (4):545-51.

31. Kumagai Koji, SakamotoTamotsu, NakamuraKeijiro, HayanoMamoru, YamashitaEiji, OshimaShigeru. Pre-procedural prediction of termination of persistent atrial fibrillation by catheter ablation as an indicator of reverse remodeling of the left atrium. Circ. J. 2013;77 (6):1416-23.

32. Combes Stéphane,JacobSophie, CombesNicolas, KaramNicole, ChaumeilArnaud, Guy-MoyatBenoit, TreguerFrédéric, DeplagneAntoine, BovedaSerge, MarijonEloi, AlbenqueJean-Paul. Predicting favourable outcomes in the setting of radiofrequency catheter ablation of long-standing persistent atrial fibrillation: a pilot study assessing the value of left atrial appendage peak flow velocity. Arch Cardiovasc Dis. 2013;106 (1):36-43.

33. Faustino Massimiliano, PizziCarmine, CapuzziDonato, AgricolaTullio, CostaGrazia Maria, FlaccoMaria Elena, MarzuilloCarolina, NoccioliniManuela, CapassoLorenzo, ManzoliLamberto. Impact of atrial fibrillation termination mode during catheter ablation procedure on maintenance of sinus rhythm. Heart Rhythm. 2014;11 (9):1528-35.

34. Drewitz Imke, WillemsStephan, SalukheTushar V, StevenDaniel, HoffmannBoris A, ServatiusHelge, BockKarsten, AydinMuhammet Ali, WegscheiderKarl, MeinertzThomas, RostockThomas. Atrial fibrillation cycle length is a sole independent predictor of a substrate for consecutive arrhythmias in patients with persistent atrial fibrillation. Circ Arrhythm Electrophysiol. 2010;3 (4):351-60.

35. Khurram Irfan M, HabibiMohammadali, Gucuk IpekEsra, ChrispinJonathan, YangEunice, FukumotoKotaro, DewireJane, SpraggDavid D, MarineJoseph E, BergerRonald D, AshikagaHiroshi, RickardJack, ZhangYiyi, ZipunnikovVadim, ZimmermanStefan L, CalkinsHugh, NazarianSaman. Left Atrial LGE and Arrhythmia Recurrence Following Pulmonary Vein Isolation for Paroxysmal and Persistent AF. JACC Cardiovasc Imaging. 2016;9 (2):142-8.

36. O’Neill Mark D, WrightMatthew, KnechtSébastien, JaïsPierre, HociniMélèze, TakahashiYoshihide, JönssonAnders, SacherFrédéric, MatsuoSeiichiro, LimKang Teng, ArantesLeonardo, DervalNicolas, LelloucheNicholas, NaultIsabelle, BordacharPierre, ClémentyJacques, HaïssaguerreMichel. Long-term follow-up of persistent atrial fibrillation ablation using termination as a procedural endpoint. Eur. Heart J. 2009;30 (9):1105-12.

37. Schreiber Doreen, RostockThomas, FröhlichMax, SultanArian, ServatiusHelge, HoffmannBoris A, LükerJakob, BernerImke, SchäfferBenjamin, WegscheiderKarl, LeziusSusanne, WillemsStephan, StevenDaniel. Five-year follow-up after catheter ablation of persistent atrial fibrillation using the stepwise approach and prognostic factors for success. Circ Arrhythm Electrophysiol. 2015;8 (2):308-17.

38. Scherr Daniel, KhairyPaul, MiyazakiShinsuke, Aurillac-LavignolleValerie, PascalePatrizio, WiltonStephen B, RamoulKhaled, KomatsuYuki, RotenLaurent, JadidiAmir, LintonNick, PedersenMichala, DalyMatthew, O’NeillMark, KnechtSebastien, WeerasooriyaRukshen, RostockThomas, ManningerMartin, CochetHubert, ShahAshok J, YeimSunthareth, DenisArnaud, DervalNicolas, HociniMeleze, SacherFrederic, HaissaguerreMichel, JaisPierre. Five-year outcome of catheter ablation of persistent atrial fibrillation using termination of atrial fibrillation as a procedural endpoint. Circ Arrhythm Electrophysiol. 2015;8 (1):18-24.

39. Tilz Roland Richard, RilligAndreas, ThumAnna-Maria, AryaAnita, WohlmuthPeter, MetznerAndreas, MathewShibu, Yoshiga Yasuhiro, WissnerErik, KuckKarl-Heinz, OuyangFeifan. Catheter ablation of long-standing persistent atrial fibrillation: 5-year outcomes of the Hamburg Sequential Ablation Strategy. J. Am. Coll. Cardiol. 2012;60 (19):1921-9.

40. Rostock Thomas, SalukheTushar V, StevenDaniel, DrewitzImke, HoffmannBoris A, BockKarsten, ServatiusHelge, MüllerleileKai, SultanArian, GosauNils, MeinertzThomas, WegscheiderKarl, WillemsStephan. Long-term single- and multiple-procedure outcome and predictors of success after catheter ablation for persistent atrial fibrillation. Heart Rhythm. 2011;8 (9):1391-7.

41. Komatsu Yuki, TaniguchiHiroshi, MiyazakiShinsuke, NakamuraHiroaki, KusaShigeki, UchiyamaTakashi, KakitaKen, KakutaTsunekazu, HachiyaHitoshi, IesakaYoshito. Impact of atrial fibrillation termination on clinical outcome after ablation in relation to the duration of persistent atrial fibrillation. Pacing Clin Electrophysiol. 2012;35 (12):1436-43.

42. Yoshida Kentaro, RabbaniAmir B, OralHakan, BachDavid, MoradyFred, ChughAman. Left atrial volume and dominant frequency of atrial fibrillation in patients undergoing catheter ablation of persistent atrial fibrillation. J Interv Card Electrophysiol. 2011;32 (2):155-61.

43. Rostock Thomas, SalukheTushar V, HoffmannBoris A, StevenDaniel, BernerImke, MüllerleileKai, TheisCathrin, BockKarsten, ServatiusHelge, SultanArian, WillemsStephan. Prognostic role of subsequent atrial tachycardias occurring during ablation of persistent atrial fibrillation: a prospective randomized trial. Circ Arrhythm Electrophysiol. 2013;6 (6):1059-65.

44. Miyazaki Shinsuke, TaniguchiHiroshi, KomatsuYuki, UchiyamaTakashi, KusaShigeki, NakamuraHiroaki, HachiyaHitoshi, IsobeMitsuaki, HiraoKenzo, IesakaYoshito. Sequential biatrial linear defragmentation approach for persistent atrial fibrillation. Heart Rhythm. 2013;10 (3):338-46.

45. Ammar S, HesslingG, PaulikM, ReentsT, DillierR, BuiattiA, SemmlerV, KolbC, HallerB, DeisenhoferI. Impact of baseline atrial fibrillation cycle length on acute and long-term outcome of persistent atrial fibrillation ablation. J Interv Card Electrophysiol. 2014;41 (3):253-9.

46. Di Biase Luigi, BurkhardtJ David, MohantyPrasant, SanchezJavier, MohantySanghamitra, HortonRodney, GallinghouseG Joseph, BaileyShane M, ZagrodzkyJason D, SantangeliPasquale, HaoSteven, HongoRichard, BeheirySalwa,ThemistoclakisSakis, BonsoAldo, RossilloAntonio, CorradoAndrea, RavieleAntonio, Al-AhmadAmin, WangPaul, CummingsJennifer E, SchweikertRobert A, PelargonioGemma, Dello RussoAntonio, CasellaMichela, SantarelliPietro, LewisWilliam R, NataleAndrea. Left atrial appendage: an underrecognized trigger site of atrial fibrillation. Circulation. 2010;122 (2):10918.

47. Rillig Andreas, TilzRoland R, LinTina, FinkThomas, HeegerChristian-H, AryaAnita, MetznerAndreas, MathewShibu, WissnerErik, MakimotoHisaki, WohlmuthPeter, KuckKarl-Heinz, OuyangFeifan. Unexpectedly High Incidence of Stroke and Left Atrial Appendage Thrombus Formation After Electrical Isolation of the Left Atrial Appendage for the Treatment of Atrial Tachyarrhythmias. Circ Arrhythm Electrophysiol. 2016;9 (5):e003461.

48. Corradi Domenico, CallegariSergio, BenussiStefano, NascimbeneSimona, PastoriPaolo, CalviSimone, MaestriRoberta, AstorriEttore, PapponeCarlo, AlfieriOttavio. Regional left atrial interstitial remodeling in patients with chronic atrial fibrillation undergoing mitral-valve surgery. Virchows Arch. 2004;445 (5):498-505.

49. Muellerleile Kai, GrothMichael, StevenDaniel, HoffmannBoris A, SaringDennis, RadunskiUlf K, LundGunnar K, AdamGerhard, RostockThomas, WillemsStephan. Cardiovascular magnetic resonance demonstrates reversible atrial dysfunction after catheter ablation of persistent atrial fibrillation. J. Cardiovasc. Electrophysiol. 2013;24 (7):762-7.

50. Machino-Ohtsuka Tomoko, SeoYoshihiro, IshizuTomoko, YanakaSatomi, NakajimaHideki, AtsumiAkiko, YamamotoMasayoshi, KawamuraRyo, KoshinoYuki, MachinoTakeshi, KurokiKenji, YamasakiHiro, IgarashiMiyako, SekiguchiYukio, TadaHiroshi, AonumaKazutaka. Significant improvement of left atrial and left atrial appendage function after catheter ablation for persistent atrial fibrillation. Circ. J. 2013;77 (7):1695-704.

51. Kim Joon Bum, YangDong Hyun, KangJoon-Won, JungSung-Ho, ChooSuk Jung, ChungCheol Hyun, SongJae-Kwan, LeeJae Won. Left atrial function following surgical ablation of atrial fibrillation: prospective evaluation using dual-source 
cardiac computed tomography. Yonsei Med. J. 2015;56 (3):608-16.

52. Isobe F, KumanoH, IshikawaT, SasakiY, KinugasaS, NagamachiK, KatoY. A new procedure for chronic atrial fibrillation: bilateral appendage-preserving maze procedure. Ann. Thorac. Surg. 2001;72 (5):1473-8.

53. Lee Chee-Hoon, KimJoon Bum, JungSung-Ho, ChooSuk Jung, ChungCheol Hyun, LeeJae Won. Left atrial appendage resection versus preservation during the surgical ablation of atrial fibrillation. Ann. Thorac. Surg. 2014;97 (1):124-32.

54. Jang Sung-Won, OhYong-Seog, ShinWoo-Seung, UhmJae Sun, KimSungHwan, KimJi-Hoon, LeeMan-Young, RhoTai-Ho. Impact of left anterior line on left atrial appendage contractility in patients who underwent catheter ablation for chronic atrial fibrillation. Pacing Clin Electrophysiol. 2014;37 (2):179-87.

55. Fiala Martin, BulkováVeronika, ŠkňouřilLibor, NevřalováRenáta, TomanOndřej, JanuškaJaroslav, ŠpinarJindřich, WichterleDan. Functional improvement after successful catheter ablation for long-standing persistent atrial fibrillation. Europace. 2016.

56. Gelsomino Sandro, Van BreugelHenrica N A M, PisonLaurant, PariseOrlando, CrijnsHanry J G M, WellensFrancis, MaessenJos G, La MeirMark. Hybrid thoracoscopic and transvenous catheter ablation of atrial fibrillation. Eur J Cardiothorac Surg. 2014;45 (3):401-7.

57. Je Hyung Gon, ShumanDeborah J, AdNiv. A systematic review of minimally invasive surgical treatment for atrial fibrillation: a comparison of the Cox-Maze procedure, beating-heart epicardial ablation, and the hybrid procedure on safety and efficacy. Eur J Cardiothorac Surg. 2015;48 (4):531-40.

58. Boersma Lucas V A, CastellaManuel, van BovenWimjan, BerruezoAntonio, YilmazAlaaddin, NadalMercedes, SandovalElena, CalvoNaiara, BrugadaJosep, KelderJohannes, WijffelsMaurits, MontLluís. Atrial fibrillation catheter ablation versus surgical ablation treatment (FAST): a 2-center randomized clinical trial. Circulation. 2012;125 (1):23-30.

59. Edgerton Zachary, PeriniAlessandro Paoletti, HortonRodney, TrivediChintan, SantangeliPasquale, BaiRong, GianniCarola, MohantySanghamitra, BurkhardtJ David, GallinghouseG Joseph, SanchezJavier E, BaileyShane, LaneMaegen, DI BiaseLuigi, SantoroFrancesco, PriceJustin, NataleAndrea. Hybrid Procedure (Endo/Epicardial) versus Standard Manual Ablation in Patients Undergoing Ablation of Longstanding Persistent Atrial Fibrillation: Results from a Single Center. J. Cardiovasc. Electrophysiol. 2016;27 (5):524-30. 